\title{
Increased food contamination with mold and fungi during COVID-19 crisis
}

\author{
Narges Ansari, ${ }^{1}$ Mohadeseh Pirhadi, ${ }^{2}$ Mahsa Alikord, ${ }^{2}$ Mahmoud Bahmani, ${ }^{3}$ Parisa Sadighara, ${ }^{2}$ \\ Aliasghar Manouchehri ${ }^{4}$ \\ ${ }^{1}$ Internal Medicine Specialist, School of Medicine, Isfahan Medical Sciences (UMS), Isfahan; ${ }^{2}$ Department of \\ Environmental Health Engineering, Division of Food Safety \& Hygiene, School of Public Health, Tehran University of \\ Medical Sciences, Tehran; ${ }^{3}$ Biotechnology and Medicinal Plants Research Center, Ilam University of Medical Sciences, \\ Ilam; ${ }^{4}$ Department of Internal Medicine, Shahid Beheshti Hospital, Babol University of Medical Sciences, Babol, Iran
}

\begin{abstract}
COVID-19, known as the "novel coronavirus disease 2019", is a respiratory illness, and the causative pathogen is officially named as Sars-CoV-2, whose epidemic has spread rapidly worldwide. Thus, human-to-human transmission has reduced as people's attention to health has increased and precautionary measures have been implemented. It is known that the virus can survive on infected surfaces and hands for hours or days. It is possible to get infected by touching the contaminated surface of food packaging by customers in the grocery and then touching their own mouth, nose, or eyes. Thus, the public supposes that touching food or food packing by salesmen and buyers in the grocery can transmit the virus to the customers. Therefore, the World Health Organization encourages people to wash their hands regularly and disinfect areas where the virus can survive, such as metal and plastic surfaces. However, overwashing can cause disadvantages such as moisture penetration into food packages and increased water activity in food, which provides the conditions for fungi to grow and cause spoilage in food.
\end{abstract}

Correspondence: Aliasghar Manouchehri, Department of Internal Medicine, Shahid Beheshti Hospital, Babol University of Medical Sciences, Postal code: 47176-47745, Babol, Iran.

E-mail: drmanouchehri@yahoo.com

Key words: COVID-19; Sars-CoV-2; food safety; mycotoxins; toxic; fungal; spoilage.

Acknowledgments: The authors give their special thanks to healthcare sections and everyone who is trying to minimize the possible adverse effects of COVID-19 pandemic.

Conflict of interest: The authors declare no conflict of interest.

Received for publication: 24 May 2021.

Revision received: 21 September 2021.

Accepted for publication: 29 September 2021.

${ }^{\circ}$ Copyright: the Author(s), 2021

Licensee PAGEPress, Italy

Journal of Biological Research 2021; $94: 9874$

doi:10.4081/jbr.2021.9874

This article is distributed under the terms of the Creative Commons Attribution Noncommercial License (by-nc 4.0) which permits any noncommercial use, distribution, and reproduction in any medium, provided the original author(s) and source are credited.
Accordingly, this phenomenon would have shown significant negative effects on public health as the post-pandemic phase of COVID19. The coronavirus has had a significant impact on people's behavior in the food chain, washing and disinfecting food in these critical situations. People also quickly changed the way they bought and procured food from supermarkets to ensure food safety and eradicate the virus. Use of substances/disinfectants such as Sodium hypochlorite reduce COVID-19 from surfaces and objects or reduce viral particles from surfaces and objects. Washing and disinfecting food packaging may cause damage to products thereby reducing their shelf life and utilization. Therefore, health experts report public awareness of hands and disinfect surfaces regularly to eliminate the virus. It is also recommended to wash and disinfect hands and disinfect surfaces.

\section{Introduction}

Since late December 2019, there has been an outbreak of a novel enveloped RNA betacoronavirus called severe acute respiratory syndrome coronavirus 2 (Sars-CoV-2). The contagious disease first started in Wuhan, China, and then spread throughout the world in a short time. This virus causes coronavirus disease 2019 (COVID-19), which has become an ongoing pandemic. The novel coronavirus COVID-19 is the seventh member of the Coronaviridae family known to infect humans. ${ }^{1}$ The main symptoms of COVID-19 are fever, fatigue, dry cough, nasal congestion, runny nose, and diarrhea. Also in acute conditions of the disease are symptoms such as acute respiratory distress syndrome and viral septic shock. ${ }^{1}$

The ongoing COVID-19 pandemic is a significant health threat worldwide. ${ }^{2}$ Transmission of the coronavirus can be through droplets of cough and sneezing and close contact with a sick person or carrier. The virus can also live on surfaces and objects for hours and days, leading to disease.,4 The World Health Organization (WHO) has encouraged people in many countries to disinfect and wash food packages purchased from supermarkets, as the corona virus can survive for long periods of time on surfaces, including food packaging surfaces. ${ }^{5,6}$ The corona virus has had a significant impact on people's behavior in the food chain, washing and disinfecting food in these critical situations. People also quickly changed the way they bought and procured food from supermarkets to ensure food safety and eradicate the virus.

This paper works to demonstrate the habitual behaviors for buying food products to provide a better and more understanding of how household habits of consumption are changed after the virus spread. Although the impact of COVID-19 on short- and 
long-term food security and food safety is difficult to predict, some dangerous toxins and the presence of growth conditions for some mycotoxins increased during washing and disinfection of food. With the above objectives in mind, the current paper was designed to explain the food safety concerns in relation to the COVID-19 pandemic.

The World Health Organization states that the virus is not reproducible in food and is not transmitted through food, and that there is currently no evidence that food or food packaging is associated with the transmission of COVID-19.7,8 But precautions during food shopping may prevent viral transmission. Recently published data indicates that the COVID-19 can live on inanimate objects and surfaces such as metal, glass and plastic for up to 9 days. ${ }^{5}$ In fact, food packaging may become contaminated with COVID-19 during transport. It is possible to infect by touching the contaminated plastic surface of food packaging by customers in the grocery and then touching their own mouth, nose, or eyes. So the public supposes that touching food or food packing by salesmen and buyers in the grocery can transmit the virus to the customers. Of course, the European Food Safety Authority says there has been no evidence of the virus spreading through food or food packaging. However, given the high prevalence of coronavirus, it emphasizes adherence to health guidelines for protection against COVID-19.6,9

According to the warnings of the World Health Organization and the global prevalence of COVID-19 infection, the way people prepare and buy food has changed. ${ }^{10}$ In fact, people use substances such as Sodium hypochlorite $0.1 \%$, hydrogen peroxide $0.5 \%$ and ethanol $70 \%$ to remove or reduce COVID-19 from surfaces and objects. Also, SARS-CoV could be inactivated by UV light, thermalexposure $\left(\geq 65^{\circ} \mathrm{C}\right)$, alkaline $\mathrm{pH}(>12)$, or acidic $\mathrm{pH}(<3)$ milieu. ${ }^{6}$ Of course, washing and disinfecting food packaging may cause these detergents to enter the product, damage it, change its the shelf life, and provide conditions for the growth of molds and fungi. Also, the growth of molds and fungi in foods such as Aspergillus, Fusarium and Penicillium causes the formation of mycotoxins, includingAflatoxins, Fumonizine, Zaralenone, Dioxin zinvalenol and Ochratoxins, which will be discussed in the following types of mycotoxins in food industries. ${ }^{11}$

\section{Contamination and types of mycotoxins in dry food}

Mycotoxins are secondary toxic fungal metabolites which can contaminate crops throughout the food chain. ${ }^{12}$ They are of diverse chemical structure, varied toxicity and stable to most of food processing methods. As the consequence, mycotoxins are carried over in the food and feed chain. ${ }^{13}$ In recent years, food safety and quality have increased and attention to consumer health protection is a necessity. Fungal contamination of food leads to the production of mycotoxins at a temperature of $25-30^{\circ} \mathrm{C}$, moisture contents of above $16 \%$ with corresponding water activity of $0.70 .{ }^{14}$

\footnotetext{
Aflatoxins

Aflatoxins (AFTs)are toxic metabolites that are formed by a specific genus of molds named Aspergillus; these are Aspergillus nomicus, Aspergillus flavus, and Aspergillus parasiticus. The most common and toxic is aflatoxin B1. Improper storage conditions of food provide the conditions for their growth. Aflatoxin is a global food safety concern as recognized by the World Health Organisation (WHO $)^{15}$ which proposed the limit for all aflatoxin in food is 5$10 \mu \mathrm{g} / \mathrm{kg}$. Maximum levels for aflatoxin B1 contamination permitted
}

in human food in the United States and Europe are $20 \mathrm{ng} / \mathrm{g}$ and 4 $15 \mathrm{ng} / \mathrm{g}$, respectively, depending on the commodity. ${ }^{16}$ However, failure to comply with aflatoxin levels in the diet can lead to acute and chronic exposure that can affect human health.

Acute exposure to aflatoxins can cause liver damage, as well as liver cancer, and in chronic conditions can impair a child's growth and suppress the immune system. ${ }^{17}$

\section{Ochratoxin A}

Ochratoxin A(OTA) is mainly produced by Aspergillus ochraceus and Penicillumv errucosum and is a pentaketide derived from a dihydrocoumarin family derivative coupled to $\beta$-phenylalanine. The toxin was first identified in 1965 and spread throughout the world, especially in the Balkans, studies show that more toxicity was found to be induced by OTA, including nephrotoxicity, hepatotoxicity, teratogenicity, immunotoxicity, neurotoxicity and genotoxicity. Ocratoxin A contaminates many foods such as grapes, coffee, cocoa, and baby food. This is very worrying for human health. However, in 2007, the Joint FAO/WHO Expert Committee on Food Additives (JECFA) reset the provisional tolerable weekly intake (PTWI) down to $100 \mathrm{ng} / \mathrm{kg} \cdot \mathrm{bw} / \mathrm{week}^{18}$

\section{Fumonisins}

The fumonisins are a group of non-fluorescent mycotoxins (FB1, FB2 and FB3 being the major entities) produced primarily by Fusarium verticillioides and $F$. proliferatum. Strains vary in toxin-producing ability. They are frequently found in products such as nuts, corn, sorghum, rice, and several other cereals, which can be contaminated in the field during harvest or during storage. Studies show that Fumonisin in cereals causes esophageal cancer, leukoence, phalomacia in equine species and rabbits, pulmonary edema and hydrothorax in pigs and hepatotoxic, carcinogenic and apoptosis (programmed cell death) effects in the liver of rats. ${ }^{19}$ The maximum levelfor fumonisins contamination permitted in human foods and animal feeds in U.S. Food and Drug Administration guidelines is $4 \mu \mathrm{g} / \mathrm{g} .{ }^{20}$

\section{Zearalenones}

Zearalenone is a mycotoxin produced mainly by Fusarium graminerarum, F. semitectum, F. equiseti, F. culmorum, and $F$. crookwelense. Its structure, in fact, resembles $7 \mathrm{~b}$-estradiol, the main hormone produced in the human female ovary. Zearalenone would better fit the classification of a non-steroidal estrogen or a mycoestrogen. It is found in important crops such as corn, wheat, barley, sorghum and rye throughout various countries of the world. However, zearalenone can be formed at relatively cool temperatures and have led to increased levels of this mycotoxin during storage where conditions for fungal growth and mycotoxin formation were favorable. These toxins have estrogenic properties and stimulate estrus in mice and increase estrogen in pigs. ${ }^{19}$

So far, no US regulations have been imposed on the occurrence of this mycotoxin, however, the European Union states that the Dose is $200 \mu \mathrm{g} / \mathrm{kg} .{ }^{20}$

\section{Deoxynivalenol}

Deoxynivalenol (DON) is a mycotoxin belonging to the group of trichothecenes. DON, also known as vomitoxin, is produced principally by Fusarium graminearum and in some geographical areas by F. culmorum. It is found in dry and grainy foods such as wheat, oats, barley and corn. Cool and humid temperatures are suitable conditions for contamination of this toxin in food products. Consumption of DON-contaminated food causes vomiting 
syndrome and suppression of the immune system and acute liver damage in animals and weight gain suppression, anorexia and altered nutritional efficiency. In 2010, the Joint FAO/WHO Expert Committee on Food Additives (JECFA) extended it to the group of DON and its acetyl derivatives (3-acetyl-DON (3-Ac-DON) and 15-acetyl-DON (15-Ac-DON) and also derived an Acute Reference Dose (ARfD) at $8 \mu \mathrm{g} / \mathrm{kg}$ b.w..$^{20,21}$

\section{Trichothecenes}

Trichothecenes are a group of mycotoxins mainly produced byfungi species such as Fusarium, Myrothecium, and Stachybotrys. Consumers are particularly concerned over the toxicity and food safety of trichothecenes and their metabolites from food-producing animals. ${ }^{22} \mathrm{~T}-2$ toxin can be frequently observed in cereals, particularly in oats, barley, wheat, corn, rice, sorghum, beans, and animal feeds, especially in the cooler and humid regions. ${ }^{23}$ Side effects of this toxin in humans and animals include hemorrhaging, diarrhea, skin lesion, emesis, feed refusal, weight loss, leucopenia, immunosuppression, oxidative stress, radiomimetic injury to tissues, and death. ${ }^{22,24}$ However, no regulations are present for T-2 toxin in commodities or any other products. ${ }^{20}$

\section{Conclusions}

Since WHO announced the outbreak of COVID-19, international authorities have repeatedly stated that the disease is a viral infection that can remain on the surface for a long time. Therefore, health experts recommend that people wash their hands and surfaces regularly to eliminate the virus. Excessive washing of plastic food packages, of course, may lead to the penetration of water and detergents into the food packaging, and by creating a humid environment, conditions are provided for the growth of mold. It is estimated that the inflow of water and detergents into food is increasing. Consequently, it is possible to increase spoilage in dry foods that have been around for a long time. Therefore, the authors report public awareness of the penetration of detergents into food and the growth of fungi and food spoilage.

\section{References}

1. Wu CY, Yu XY, Ma AH, et al. Coronavirus disease 19 with gastrointestinal symptoms as initial manifestations: a case report. J Int Med Res 2020;48:0300060520952256.

2. Sivakorn C, Luvira V, Muangnoicharoen S, et al. Case report: walking pneumonia in novel coronavirus disease (COVID-19): mild symptoms with marked abnormalities on chest imaging. AmJ Tropical MedHygiene 2020;102:940-2.

3. Cai J, Huang J, Gamber M, et al. Indirect virus transmission in cluster of COVID-19 cases, Wenzhou, China. Emerg Infect Dis 2020;26:1343-5.

4. Carraturo F, Del Giudice C, Morelli M, et al. Persistence of SARS-CoV-2 in the environment and COVID-19 transmission risk from environmental matrices and surfaces. Environ Pollut 2020;265:115010.
5. Marquès M, Domingo JL. Contamination of inert surfaces by SARS-CoV-2: Persistence, stability and infectivity. A review. Environ Res 2020;193:110559.

6. Pressman P, Naidu AS, ClemensR. COVID-19 and food safety: risk management and future considerations. Nutrition Today 2020;55:125-8

7. World Health Organization \& Food and Agriculture Organization of the United Nations. ( $\square 2020) \square$. COVID-19 and food safety: guidance for food businesses: interim guidance, 07 April 2020. World Health Organization. Available from: https://apps.who.int/iris/handle/10665/331705

8. Desai AN, Aronoff DM. Food safety and COVID-19. JAMA 2020;323:1982.

9. Galanakis CM. The food systems in the era of the coronavirus (COVID-19) pandemic crisis. Foods 2020;9:523.

10. Cappelli A, Cini E. Will the COVID-19 pandemic make us reconsider the relevance of short food supply chains and local productions? Trends Food Sci Technol 2020;99:566.

11. Udomkun P, Nimo Wiredu A, Nagle M, et al. Mycotoxins in Sub-Saharan Africa: Present situation, socio-economic impact, awareness, and outlook. Food Control 2017;72:110-22.

12. Ezekiel CN, Warth B, Ogara IM, et al. Mycotoxin exposure in rural residents in northern Nigeria: a pilot study using multiurinary biomarkers. Environ Int 2014;66:138-45.

13. Probst C, Bandyopadhyay R, Cotty P. Diversity of aflatoxinproducing fungi and their impact on food safety in sub-Saharan Africa. Int J Food Microbiol 2014;174:113-22.

14. Thanushree M, Sailendri D, Yoha JKS, et al. Mycotoxin contamination in food: An exposition on spices. Trends Food Sci Technol 2019;93:69-80.

15. Nazir A, Kalim I, Sajjad M, et al. Prevalence of aflatoxin contamination in pulses and spices in different regions of Punjab. Chem Int 2019;5:274-80.

16. Simpson AT. Buying and eating in the dark: Can the Food and Drug Administration require mandatory labeling of genetically engineered foods. Alliance For Bio-Integrity v. Shalala, et al., 116 F. Supp. 2d 166 (2000). Temp Envtl L Tech J 2000;19:225.

17. Cardwell KF, Henry SH. Risk of exposure to and mitigation of effect of aflatoxin on human health: a West African example. J Toxicol 2004;23:217-47.

18. Zhu L, Zhang B, Dai Y, et al. A review: epigenetic mechanism in ochratoxin a toxicity studies. Toxins 2017;9:113.

19. da Rocha MEB, Oliveira Freire FDC, Maia FEF, et al. Mycotoxins and their effects on human and animal health. Food Control 2014;36:159-65.

20. Richard JL. Some major mycotoxins and their mycotoxicoses-An overview. Int J Food Microbiol 2007;119:3-10.

21. Authority EFS. Deoxynivalenol in food and feed: occurrence and exposure. EFSA J 2013;11:3379.

22. Wu Q, Dohnal V, Huang L, et al. Metabolic pathways of trichothecenes. Drug metabolism reviews 2010;42:250-67.

23. Chen P, Xiang B, Shi H, et al. Recent advances on type A trichothecenes in food and feed: Analysis, prevalence, toxicity, and decontamination techniques. Food Control 2020;118: 107371 .

24. Salahvarzi S, Shakib P, Pirhadi M, et al. Helicobacter phytotherapy: Medicinal plants affecting Helicobacter Pylori infection in Iran. Plant Biotechnol Persa 2020;2:38-42. 